\title{
Criminologie
}

\section{Les auteurs de vols à main armée à Montréal : une typologie empirique}

\section{Sylvie Bellot}

Volume 18, numéro 2, 1985

Le vol à main armée à Montréal

URI : https://id.erudit.org/iderudit/017215ar

DOI : https://doi.org/10.7202/017215ar

Aller au sommaire du numéro

Éditeur(s)

Les Presses de l'Université de Montréal

ISSN

0316-0041 (imprimé)

1492-1367 (numérique)

Découvrir la revue

Citer cet article

Bellot, S. (1985). Les auteurs de vols à main armée à Montréal : une typologie empirique. Criminologie, 18(2), 35-45. https://doi.org/10.7202/017215ar
Résumé de l'article

This article presents a typology of armed robbers in Montreal, the result of a study of 39 authors of armed robbery. After a brief outline of the theoretical and methodological model used in this study, each type of armed robber is presented as follows : 1 ) the multireci-divist who is characterized by a large number of armed robberies, 2) the professional whose criminal activity is planned and organized, 3) the intensive robber who commits armed robberies for a very short period of time and 4) the occasional robber who commits very few armed robberies but many other crimes. 
This article presents a typology of armed robbers in Montreal, the result of a study of 39 authors of armed robbery. After a brief outline of the theoretical and methodological model used in this study, each type of armed robber is presented as follows : 1) the multirecidivist who is characterized by a large number of armed robberies, 2) the professional whose criminal activity is planned and organized, 3) the intensive robber who commits armed robberies for a very short period of time and 4) the occasional robber who commits very few armed robberies but many other crimes.

\section{LE VOLEUR À MAIN ARMÉE, UN SPÉCIALISTE AUX TECHNIQUES SOPHISTIQUÉES OU UN AMATEUR QUI IMPROVISE?}

Cette question a fait l'objet de bon nombre de recherches aux États-Unis au cours des dernières décennies et il serait trop long d'en faire la revue dans cet article. Néanmoins, il nous paraît important de présenter quelques idées qui se dégagent de ces travaux, principalement en ce qui a trait à la spécialisation et au professionnalisme.

Concernant la spécialisation, la plupart des études récentes infirment en général cette assertion et révèlent que les voleurs à main armée ont des activités délictuelles plutôt diversifiées, ne se limitant pas strictement au vol à main armée. Ainsi, ils ont à leur actif de nombreux délits tels que des vols à main armée bien entendu, mais également des vols par effraction, des vols de véhicule à moteur, des vols simples, parfois des fraudes ou encore des délits reliés aux drogues, par exemple trafic et possession.

Quant au professionnalisme, qui se traduit essentiellement par des techniques de préparation et d'exécution du délit assez sophistiquées, il apparaît qu'il varie énormément selon les individus, les uns planifiant le vol à main armée de façon très détaillée au point

*Sylvie Bellot, agent de recherche, Centre international de criminologie comparée, Université de Montréal. 
de prévoir un avocat ou un médecin dans l'éventualité d'un échec et les autres organisant le khold up» plutôt sommairement lorsqu'ils n'improvisent pas carrément.

Ces résultats mettent finalement en évidence le fait que la catégorie «voleurs à main armée» n'est pas homogène en soi puisqu'elle réfère à des individus aux comportements très divers, tout au moins aux États-Unis. Qu'en est-il à Montréal? La vague de vols à main armée enregistrés au cours des années 70 a en effet suscité l'inquiétude mais aussi de nombreuses interrogations quant aux auteurs de ces vols : qui sont-ils? Quelles sont leurs motivations? Sont-ils spécialistes du vol à main armée? etc. ... Pour tenter d'apporter des réponses à ces questions, dans le cadre d'un programme de recherche sur le vol à main armée un volet consacré à l'étude des auteurs de vols à main armée fut mis sur pied.

\section{UNE ÉTUDE DES AUTEURS DE VOLS À MAIN ARMÉE À MONTRÉAL ${ }^{1}$}

Entreprise à l'été 1982, cette étude des auteurs de vols à main armée à Montréal s'est terminée en avril 1984 et a permis d'élaborer une typologie des voleurs à main armée. Avant de présenter cette dernière, nous allons toutefois donner un aperçu de l'organisation de la recherche, c'est-à-dire de la problématique et de la méthodologie qui s'y rattachent.

En débutant cette étude, l'objectif était de mieux connaître le vol à main armée et les raisons pour lesquelles on s'y engage. on y persévère et or l'abandonne. À cette fin, il fut décidé d'articuler la problématique essentiellement autour de trois thèmes, soit les activités délinquantes, les motivations et l'expérience pénale. En outre, considérant que les protagonistes étaient sans doute les plus aptes à expliquer leurs actes, un protocole d'entrevue, composé essentiellement de questions ouvertes fut élaboré et administré

1. Cette étude des auteurs de vols à main armée était dirigée par Maurice Cusson, professeur titulaire à l'Ecole de criminologie, Université de Montréal. Elle a donné lieu à la publication de trois rapports techniques (voir Bellot, 1983; Dionne, 1984 et Pinsonneault, 1984) et d'un rapport final (voir Bellot et al., 1984). L'auteur tient à remercier Maurice Cusson pour sa supervision efficace et Michèle Dionne et Pierre Pinsonneault pour leur collaboration. 
à une quarantaine de volontaires recrutés en milieu pénitentiaire. Ces derniers avaient toutefois été classés auparavant en fonction du nombre de vols à main armée inscrits à leur dossier officiel. Le groupe de sujets comprenait ainsi 27 voleurs à main armée dits multirécidivistes (ayant plus de trois condamnations officielles pour vol à main armée) et 12 dits occasionnels (ayant au moins une condamnation officielle pour vol à main armée et trois tout au plus), soit un total de 39 individus.

Pour en revenir au questionnaire, il comprenait plusieurs sections. La première était composée de questions d'ordre général telles que l'âge, le degré de scolarité, les différents emplois occupés par le sujet, son statut, etc. ... La seconde portait plus spécifiquement sur l'engagement dans le vol à main armée et l'abandon s'il y avait lieu (motivations qui ont guidé le choix du vol à main armée, comparaison de ce dernier avec d'autres types d'activités criminelles, etc. ...). La troisième section traitait surtout du cheminement suivi par le sujet depuis son premier délit jusqu'au moment de l'entrevue, notamment de ses premiers contacts avec le système pénal, de ses diverses expériences, en milieu carcéral, de ses valeurs actuelles et de la pratique acquise dans le domaine du vol à main armée, entre autres par la description de deux vols à main armée, l'un réussi et l'autre raté. Dans la quatrième section, il était surtout question des activités délictuelles et des expériences institutionnelles du sujet lorsqu'il avait moins de 18 ans. Enfin, la dernière partie était un questionnaire de délinquance autorévélée, inspiré de celui de Petersilia et al. (1978).

Les données quantitatives ont été comptabilisées; quant aux informations qualitatives, la méthode d'analyse employée a consisté à regrouper les réponses des sujets de façon thématique et à faire ressortir les opinions et idées les plus souvent mentionnées tout en tenant compte de la diversité de celles-ci.

Les résultats ont révélé un certain nombre de points communs entre les voleurs à main armée interrogés, notamment au niveau des motivations, du genre de vie qu'ils mènent et de leur expérience en milieu carcéral, mais également des différences.

En premier lieu, on peut parler de différences en ce qui concerne la délinquance des sujets et ce, tant quantitativement que qualitativement. En effet, certains ont commis cinq à dix vols à main armée et n'ont jamais fait d'autres types de délits, d'autres 
par contre ont très peu de vols à main armée à leur actif mais sont plus ou moins spécialisés dans le vol par effraction, la fraude, la vente de drogue, etc., et ont essayé à peu près tous les genres de vols possibles (vol à la tire, vol d'automobile). D'autres encore sont très impliqués dans le vol à main armée et en ont commis un nombre impressionnant sans pour autant négliger d'autres activités illégales. Finalement, l'expérience délictuelle des sujets s'avère très variée et il est impossible de dégager un seul modèle de cheminement pour tous les voleurs que nous avons rencontrés.

Une deuxième différence concerne les périodes d'inactivité dans le crime. Ainsi, certains s'adonnent au vol à main armée sur une période de temps très limitée puis abandonnent ensuite toute activité illégale. D'autres, à l'inverse, commencent très tôt leur agir délinquant et connaissent très peu de périodes d'inactivité criminelle, celles-ci étant par ailleurs de courte durée (quelques semaines ou quelques mois, au plus). Certains par contre sont très actifs durant un certain temps puis cessent de commettre des délits pendant plusieurs mois, plusieurs années, pour reprendre finalement par après. Là encore, le cheminement n'est pas le même pour tous.

L'étendue de la carrière dans le vol à main armée permet également de distinguer plusieurs groupes de voleurs. En effet, pour quelques-uns elle est très courte et s'avère de quelques semaines ou quelques mois, pour d'autres elle s'étend sur plusieurs années et ne semble pas sur le point de se terminer.

Par ailleurs, nous avons pu constater des différences importantes entre les sujets en ce qui concerne l'exécution du vol à main armée proprement dit, notamment au niveau de la planification, ainsi celle-ci peut être minutieuse et, à l'inverse, inexistante.

Enfin, les revenus obtenus grâce au vol à main armée et l'utilisation de cet argent varient également selon les individus.

Toutes ces différences, combinées les unes aux autres, mettent en évidence l'existence de quatre types de voleurs à main armée parmi les sujets :

- le multirécidiviste;

- le professionnel;

- 1'intensif;

- l'occasionnel. 
Avant de décrire précisément chacune de ces catégories, il convient d'ajouter que les sujets interrogés se sont distribués comme suit selon la typologie, 11 ont été classés multirécidivistes, 16 professionnels, 4 intensifs et 8 occasionnels.

\section{LE VOLEUR À MAIN ARMÉE MULTIRÉCIDIVISTE}

Le voleur à main armée multirécidiviste s'engage généralement très tôt dans la délinquance. Ainsi, il commet son premier délit en moyenne à l'âge de 12 ans et se fait arrêter pour la première fois vers 14 ans. Ses débuts sont surtout marqués par des vols simples, du vandalisme et des vols par effraction. Arrivé vers 17-18 ans, il exécute souvent son premier vol à main armée, c'est alors le commencement d'une "carrière de voleur à main armée» qui s'étendra sur plusieurs années. Bien qu'on observe des variations importantes dans la durée de cette carrière selon les individus, la moyenne est de 7 à 8 ans. Tout en s'adonnant essentiellement au vol à main armée, il est fréquent que le multirécidiviste continue de commettre d'autres délits de temps à autre, tels que des vols par effraction, de la vente de drogue ou encore des vols d'automobile (la plupart des sujets préférant utiliser une voiture volée pour l'exécution d'un vol à main armée). Si environ la moitié des multirécidivistes n'ont pas voulu nous dire combien de vols à main armée ils avaient commis, les chiffres dont nous disposons permettent de croire que le nombre varie en moyenne de 20 à 50. Quant aux autres délits, c'est un chiffre à peu près semblable dans l'ensemble. Il arrive néanmoins que, pour certains, ces limites soient amplement dépassées.

En ce qui concerne le vol à main armée, la façon d'opérer du multirécidiviste est la suivante. Après avoir repéré une cible éventuelle, il procède à une évaluation sommaire des risques et de l'argent à gagner. Si l'emplacement de l'endroit à attaquer facilite la fuite, il décide alors de passer à l'action. Ainsi, le repérage des lieux est généralement rapide et prend peu de temps, soit quelques minutes ou quelques heures, à la limite un ou deux jours avant. Il importe toutefois de préciser que le sujet dispose assez souvent d'armes, de déguisements et d'une voiture volée, ce qui simplifie la préparation du vol, le choix de la cible étant alors la dernière chose à accomplir. Lorsqu'il exécute le vol proprement dit, le multirécidiviste est presque toujours déguisé mais, surtout, il est bien armé. Dans $80 \%$ des cas, il s'agit d'une arme à feu chargée 
et le voleur n'hésite pas à tirer s'il se sent menacé. Ainsi, sur vingt-deux vols qui nous ont été racontés, les armes ont été utilisées dans cinq cas. Toutefois, il n'y a pas toujours eu de blessés, les coups étant tirés parfois au plafond et au mur. Par ailleurs, le multirécidiviste a recours à la prise d'otages à l'occasion, ce qui traduit une certaine détermination.

Finalement, le vol à main armée s'avère une activité rentable. En effet, il demande peu de préparation, s'exécute rapidement et rapporte à son auteur entre $500 \$$ et $5000 \$$ en moyenne, chaque fois. De plus, comme le sujet ne répugne pas à employer la violence au besoin, le moyen apparaît vraiment facile et idéal. L'argent gagné grâce au vol à main armée est, néanmoins dépensé très rapidement. Ainsi, bon nombre s'achètent des drogues, de l'alcool, vont dans les clubs, etc., d'autres se paient une automobile et partent en voyage, etc., bref, l'argent est surtout employé à rendre agréable le moment présent, son utilisation semble plutôt irrationnelle. Cette constatation reliée au fait que le délit est peu planifié nous amène à dire du multirécidiviste qu'il est essentiellement tourné vers le présent et se soucie peu de l'avenir. C'est ce que Cusson (1981) appelle le "présentisme». Par ailleurs, ceci permet peut-être d'expliquer l'importance de la récidive, la gravité des délits et la rareté des périodes d'inactivité criminelle chez le multirécidiviste.

\section{LE VOLEUR A MAIN ARMÉE PROFESSIONNEL}

Avant de dresser le portrait du voleur à main armée professionnel, il importe de préciser qu'en dehors de la délinquance officielle, nous avons peu d'informations sur l'ensemble de ses activités criminelles. En effet, un tiers des sujets dits professionnels ont refusé de répondre au questionnaire de délinquance révélée et un tiers ont fourni des réponses très évasives. De ce fait, nos commentaires, qui s'appuient sur les renseignements obtenus, ne reflètent qu'une partie de la réalité.

C'est généralement à l'adolescence que le futur «voleur à main armée professionnel» s'engage dans la délinquance. Ainsi, il commet son premier délit à l'âge de 13 ans. Le contact avec le système judiciaire se produira un peu plus tard puisque c'est en moyenne à 16 ans qu'il est arrêté pour la première fois. Enfin, c'est vers 17 ans qu'il fait ses premières armes dans le vol à main armée. Bien qu'ayant peu d'informations sur cette période, on peut ima- 
giner que le sujet a eu le temps de s'initier à différents types de vols auparavant tels que le vol par effraction, le vol d'automobiles, etc.

La carrière dans le vol à main armée est relativement longue puisqu'elle s'étend sur plusieurs années et dure en moyenne 11 à 12 ans. Elle n'empêche toutefois pas le professionnel de poursuivre simultanément d'autres activités illégales comme la vente de drogue pour certains, le vol par effraction (vol de coffre-fort) pour d'autres, ou encore le vol d'automobiles. En effet, si le vol à main armée s'avère un moyen intéressant de faire de l'argent, il en existe d'autres également et le professionnel n'hésite pas à y recourir si l'enjeu en vaut la peine. Il procède néanmoins à une évaluation des risques avant de s'engager.

En ce qui a trait au vol à main armée proprement dit, le professionnel se différencie des autres types de voleurs du fait qu'il planifie en détail le vol qu'il veut commettre. La préparation d'un «coup» s'étend fréquemment sur plusieurs semaines voire même plusieurs mois. Le repérage des lieux s'effectue très minutieusement, l'auteur est alors parfois accompagné d'un ou deux complices; après avoir choisi la cible, ils étudient sa situation géographique afin d'organiser la fuite efficacement, il arrive aussi qu'ils prévoient un autre plan pour fuir au cas où le premier échouerait. Ils étudient également les caractéristiques physiques de l'endroit (portes d'entrée et de sortie, visibilité des lieux. nombre de caisses, situation du coffre, etc.) et observent les allées et venues des personnes fréquentant l'endroit en question (clients, habitués, employés, etc.). Pour planifier plus facilement le vol, il est fréquent que l'auteur loue un appartement à proximité de la cible, cela lui permet aussi d'être moins remarqué dans le secteur.

Pour exécuter le vol à main armée, le professionnel est généralement muni d'une arme à feu chargée; le plus souvent il s'agit d'un révolver ou d'un pistolet (58\% des armes utilisées), d'un fusil ou d'une carabine $(25 \%)$, ou au besoin d'une mitraillette $(17 \%)$. Il utilise parfois ses armes, toutefois, pour les sujets rencontrés, cela s'est produit moins souvent que pour les multirécidivistes. Il est cependant impossible de dire si cette manière d'agir est représentative des professionnels. Enfin, il arrive que le professionnel ait recours à la prise d'otages pour obtenir ce qu'il veut, ainsi quatre des sujets (sur un total de 16) ont treize séquestrations à leur actif, ce qui est tout de même significatif. 
Bien qu'il ne réussisse pas toujours son coup, le professionnel semble tirer des revenus appréciables du vol à main armée. Ainsi, le montant d'argent rapporté chaque fois varie généralement entre $1000 \$$ et $5000 \$$, plusieurs déclarent néanmoins avoir obtenu des sommes beaucoup plus considérables telles que $10000 \$$, $42000 \$$ et $86000 \$$. Même s'il fait beaucoup d'argent rapidement, le professionnel semble avoir une notion de l'argent très différente du multirécidiviste. Il en connaît la valeur et ne cherche pas à le dépenser inutilement. Ainsi, une bonne partie de l'argent gagné grâce au vol à main armée lui permet de payer ses dettes et ses dépenses quotidiennes (linge, nourriture, ...) de s'acheter une automobile, du mobilier, etc. Pour le reste, le professionnel s'avère très prévoyant. Ainsi, il profite souvent de cet argent pour régler d'avance plusieurs mois de loyer, il essaie également de le faire fructifier (par le biais de transactions plus ou moins légales, telles que, par exemple, achat et vente de drogue). Enfin, chose plutôt surprenante, il en dépose une partie dans un ou plusieurs comptes en banque.

Finalement, le professionnel connaît quelques périodes d'inactivité criminelle qui peuvent durer plusieurs mois et même plusieurs années pour certains. On peut imaginer que les revenus tirés des activités illégales sont assez importants pour lui permettre de vivre en toute tranquillité, "retiré des affaires», pour un certain temps. Quelques-uns déclarent néanmoins qu'ils sont toujours actifs et qu'il leur est impossible d'arrêter puisqu'ils appartiennent à un «milieu» où tout le monde s'adonne à des activités illégales.

En fin de compte, c'est dans sa vision à long terme que le professionnel s'oppose au multirécidiviste. En effet, alors que ce dernier est essentiellement axé sur la satisfaction immédiate de ses besoins, le professionnel est beaucoup plus tourné vers l'avenir. La planification de ses délits et l'utilisation de l'argent qu'il gagne témoignent très bien de cette préoccupation.

En terminant, il est important de rappeler que le peu d'informations obtenues des professionnels sur leurs activités délictuelles laisse la voie ouverte à toutes sortes de suppositions et soulève bon nombre d'interrogations quant à la véritable nature et à l'ampleur de ces activités.

\section{LE VOLEUR À MAIN ARMÉE \&INTENSIF»}

Le voleur à main armée intensif est généralement un individu qui n'a aucun ou très peu d'antécédents judiciaires et qui décide 
un beau jour de commettre un vol à main armée. Son entrée dans le vol à main armée est assez tardive puisqu'il a en moyenne $\mathbf{2 5}$ ans à ce moment. Sa carrière est néanmoins très courte et s'étend sur quelques semaines ou quelques mois au plus. Durant cette période, il effectue cinq à dix «hold up» avant de se faire arrêter.

En ce qui a trait au vol à main armée comme tel, la planification est faible ou modérée. En effet, l'intensif prend peu de temps pour préparer le délit, parfois quelques heures ou bien un ou deux jours. Cela consiste essentiellement à organiser la fuite assez sommairement et à trouver une arme et un déguisement. Pour exécuter le vol, l'intensif est souvent accompagné d'un complice, ils sont tous deux déguisés et les armes employées sont des armes à feu chargées dans la moitié des cas (autrement, il s'agit d'imitations). Celles-ci ne servent toutefois qu'à menacer et ne sont pas véritablement utilisées.

L'intensif ne tire pas des revenus extraordinaires du vol à main armée puisque les gains obtenus varient entre $150 \$$ et $1400 \$$ en moyenne (pour les sujets rencontrés). Par ailleurs, cet argent est dépensé différemment selon les individus, ainsi pour les uns il est destiné à régler les dépenses quotidiennes, pour les autres il permet de gager et jouer, ou encore d'aller dans les clubs. Bref, il n'est pas investi et disparaît rapidement.

Finalement, le voleur à main armée intensif abandonne rapidement le vol à main armée, son expérience s'est en effet soldée par un échec puisqu'il est souvent condamné à plusieurs années d'incarcération et qu'il n'a pas fait fortune. Il serait toutefois présomptueux d'affirmer qu'il ne récidivera jamais, cela dépendra, probablement, des situations et des circonstances auxquelles il sera confronté.

\section{LE VOLEUR À MAIN ARMÉE OCCASIONNEL}

Le voleur à main armée occasionnel est peut être un occasionnel dans le vol à main armée mais n'en demeure pas moins un délinquant multirécidiviste. En effet, il commence à commettre des délits dès l'âge de 13 ans et se fait arrêter pour la première fois aux alentours de 15, 16 ans en moyenne. Il a généralement une très bonne expérience délictuelle lorsqu'il décide de se lancer dans le vol à main armée. Ainsi, il a de nombreux vols simples à son actif et s'avère déjà plus ou moins spécialisé dans une branche en 
particulier, comme le vol par effraction par exemple. Il est donc déjà âgé de 20, 21 ans lorsqu'il effectue son premier «hold up». Ce dernier est déterminant, de fait lorsqu'il est réussi, il encourage l'individu à poursuivre dans la même voie.

La planification du vol n'est pas très détaillée et prend quelques heures ou quelques jours. La cible est choisie en fonction des risques qu'elle présente et de l'argent disponible et le repérage des lieux est assez sommaire dans l'ensemble. Pour exécuter le vol proprement dit, l'occasionnel n'est pas toujours déguisé (il l'est dans la moitié seulement des vols racontés) ; par contre, il est très souvent accompagné d'un complice. Côté armes, il n'est pas toujours bien équipé; ainsi, les sujets avaient une arme à feu dans la moitié des vols racontés et celle-ci n'était pas nécessairement chargée, dans les autres cas, ils avaient des imitations d'armes à feu (revolver en plastique, fusil à plombs), des couteaux (couteau de cuisine) ou encore des armes plus anachroniques telles qu'une machette. La façon de procéder de l'occasionnel témoigne donc d'un certain amateurisme en matière de vol à main armée, comparativement au professionnel ou même au multirécidiviste.

Les revenus tirés du vol à main armée varient beaucoup selon les individus mais se situent généralement entre $100 \$$ et $5000 \$$ par délit et par personne. Cet argent est «flambé» dans la drogue, les clubs, les voyages, etc., et le voleur se retrouve rapidement sans le sou.

La carrière de l'occasionnel s'étend sur quelques mois et deux ans tout au plus. En effet, il finit toujours par se faire arrêter et, après une ou deux arrestations et après avoir commis un à six vols en moyenne, il décide généralement d'abandonner. Il considère que, dans le vol à main armée, les risques sont trop élevés par rapport à l'argent qu'il peut gagner. Il préfère ainsi se recycler dans quelque chose de moins dangereux, moins violent, et tout aussi payant; certains choisissent alors la fraude, d'autres la vente de drogue, le trafic d'automobiles, etc.

Finalement, le voleur à main armée occasionnel est très opportuniste et ses périodes d'inactivité dans le crime sont plutôt rares (ou du moins très courtes, quelques semaines et au maximum quelques mois). Le vol à main armée n'est qu'un épisode dans sa carrière et il est clair qu'il n'est pas le dernier. 


\section{CONCLUSION}

Pour terminer, quelques commentaires sur la portée de cette typologie. Tout d'abord, le fait d'avoir recruté les voleurs à main armée uniquement en milieu carcéral a inévitablement introduit un biais et il est clair que le groupe de sujets sélectionnés pour cette étude n'est pas un échantillon représentatif de la population des auteurs de vols à main armée au Québec ou à Montréal.

Par contre, il importe de souligner qu'en dépit de leur nonreprésentativité, les individus interrogés totalisent à eux seuls 248 condamnations officielles pour vol à main armée ou vol avec violence. Ces chiffres sont éloquents et obligent donc à reconnaître qu'une certaine partie de la population cible a été rejointe. De ce fait, nous croyons que la typologie donne une assez bonne image de la diversité des individus qui s'adonnent au vol à main armée au Québec, notamment à Montréal.

\section{BIBLIOGRAPHIE}

BELLOT, S. (1983), Portrait du voleur à main armée occasionnel, Le vol à main armée, rapport technique no 7 , G.R.I.J., C.I.C.C., Université de Montréal.

BELLOT, S. et M. DIONNE, P. PINSONNEAULT (1984), le Vol à main armée décrit par ses auteurs, Le vol à main armée, rapport final no 2, G.R.I.J., C.I.C.C., Université de Montréal.

CUSSON, M. (1981), Délinquants pourquoi?, Ville LaSalle, Hurtubise, HMH.

DIONNE, M. (1984), Portraits de voleurs à main armée : les récidivistes et les professionnels, Le vol à main armée, rapport technique no 9 , G.R.I.J., C.I.C.C., Université de Montréal.

PETERSILIA, J. et P.W. GREENWOOD, M. LAVIN (1978), Criminal Careers of Habitual Felons, Washington, D.C., N.I.L.E.C.J., L.E.A.A., U.S. Department of Justice.

PINSONNEAULT, P. (1984), l'Abandon de la carrière criminelle, Le vol à main armée, rapport technique no 8, G.R.I.J., C.I.C.C., Université de Montréal. 